\title{
Investigation of Postoperative Recovery State of Lumbar Spinal Stenosis Patients: A Single Center Experience \\ F Altınel ${ }^{1}$, ÖA Öztürkeri ${ }^{2}$, GÖ Söylev ${ }^{3}$, C Altın $^{4}$
}

\begin{abstract}
Affiliations:
${ }^{1}$ Department of Neurosurgery, ${ }^{2}$ Neurology, ${ }^{3}$ Physical Medicine and Rehabilitation and ${ }^{4}$ Cardiology, Zübeyde Hanım Uygulama ve Araştırma Merkezi, İzmir, Turkey
\end{abstract}

\section{Correspondence:}

Dr F Altinel

Department of Neurosurgery

Faculty of Medicine

University of Baskent

6471/5 Sokak

No:7, Yalı Mahallesi

Bostanl1

Karşıyaka/İzmir

Turkey

E-mail: farukaltinel@gmail.com

Running head: Postoperative Recovery State of Lumbar Spinal Stenosis

Synopsis: Lumbar spinal stenosis is a condition that has different presentations. In this study, we aimed to investigate the changes and improvement in neurological symptoms of postoperative period lumbar spinal stenosis and lumbar disc herniation patients and discover pathognomonic radiological features. 


\begin{abstract}
Objective: In this study, we aimed to investigate the changes and improvement in neurological symptoms of postoperative period lumbar spinal stenosis (LSS) and lumbar disc herniation (LDH) patients and discover pathognomonic radiological features.
\end{abstract}

Methods: 44 LSS and 37 LDH patients with mean age of $58.70 \pm 14.58$ (24-81) that have undergone decompressive laminectomy, posterior lumbar stabilization or lumbar microdiscectomy were enrolled. Patients' demographic data, preoperative and postoperative neurological symptoms analyzed with the JOA scale were recorded and compared with the MRI results. We have measured TIP, M-AP and IF from the patients' MRI images of axial sections before a decision of surgery. We have also evaluated and graded the protrusion degrees of herniated discs in the sagittal plane of MRI. TIP, M-AP, IF values and JOA scale on were compared between LDH and LSS.

Results: All measured osteal body diameter mean values (TIP, M-AP, IF) in LSS were less than the LDH group. Only IF mean values were shown to be statistically significant $p<0.004$. Sagittal disc section measurements were not statistically significant for LSS and LDH $p<0.182$. The pre and post surgery JOA score mean values of LDH was higher than LSS group $\mathrm{p}<0.005$. Lumbar microsurgery discectomy recovery rate was higher than posterior segmental instrumentation.

Conclusion: TIP, M-AP, IF diameters decreased with age, which was the main pathological mechanism of LSS development. This suggests that the degenerative process of the narrowing spinal canal, which increases with age, is responsible for LSS presentation. LSS has a relationship with facet artropathy over other parameters.

Keywords: Decompressive Laminectomy, Lumbar Discectomy, Lumbar Spinal Stenosis 


\section{INTRODUCTION}

LSS and LDH patients have a broad spectrum of clinical symptoms, such as back pain, lower extremity paresthesia, numbness, motor disability, bladder and rectum dysfunction. The main pathology of LSS is the irreversible degenerative changes in lumbosacral nerve roots due to compression of the neural and foraminal canal. On the other hand, patients with lumbar disc herniation can also present similar clinical symptoms. The pathology predominantly is the protrusion of a herniated disc to the spinal canal. The signs and symptoms of central canal stenosis resembles cauda equina syndrome. Lateral recess stenosis can be presented by unilateral or bilateral radiculopathy, which suggests herniated disc syndrome. Lumbar spinal stenosis is one of the most frequent indications for spinal surgery of people over 60 years old and this is due to the degeneration of the spinal structure. LSS can be caused mainly by degenerative or congenital factors separately or together (5). Although congenital (developmental) stenosis is rare, degenerative (acquired) stenosis is very frequent. In the latest literature; Abbas et al. reported that in LSS patients the vertebral objects and canals have smaller diameters than normal therefore stenosis can be characterized by short pedicles and narrowing of the spinal canal dimensions. They also mentioned that both genetic factors and degenerative changes play a role in LSS development (1).

There are many studies focused on the mechanisms for the development of LSS and LDH. Ligamentum flavum hypertrophy, degenerative changes in the intervertebral discs, decrease of the anteroposterior diameter of spinal canal have been suspected so far. In this study; we investigate the diameters of the posterior bone structure (TIP, IF, M-AP). Normal and abnormal values of spinal canal diameter and diameters of its pathologies have been established by various anatomical, radiological, surgical observations. Although measurements can differ between Computed Tomography (CT) and Magnetic Resonance Imaging (MRI) techniques, a specific range to determine normal and borderline values has been used. The 
ranges used in this study on axial section of lumbar MRI are; $20-30 \mathrm{~mm}$ transverse interpedicular diameter (TIP), 15-25 mm midline anteroposterior (M-AP), 10-20 mm interfacet (IF), 3-5mm pedicular height $(\mathrm{PH}), 5-8 \mathrm{~mm}$ superior facet height $(\mathrm{SFH})$. The posterior protrusion into the spinal canal at the sagittal sections can be defined as normal range $(1 \mathrm{~mm})$, slight protrusion $(2 \mathrm{~mm})$, mild protrusion $(3 \mathrm{~mm})$, moderate protrusion $(5 \mathrm{~mm})$, and severe protrusion $(6 \mathrm{~mm})$. This is used for the grading system in order to evaluate LSS using CT and MRI measurement techniques (Figure 1).
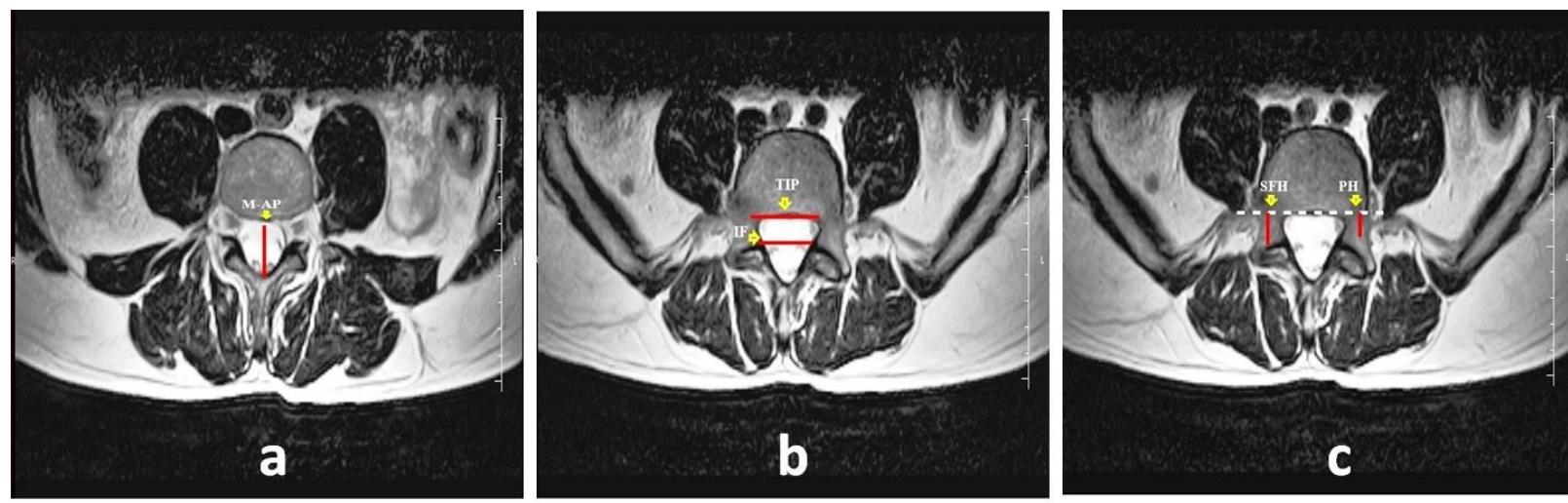

Fig 1. Measurements of axial sections of spinal canal a) M-AP; midline A-P diameter, b) TIP; transverse interpedicular diameter, IF; interfacet diameter, c) SFH; superior facet height, $\mathrm{PH}$; pedicle height.

In LSS patients, pedicule and lamina is short and thick which makes the facet slide into the midline and base of the spinal canal. Posterior bony structure has a triangular shape rather than circular and its diameter is smaller than normal. In these patients midline anteroposterior diameter is smaller than $12 \mathrm{~mm}$ and also lateral recess and foraminal diameter is smaller than $4 \mathrm{~mm}(15)$ (Figure 2). 

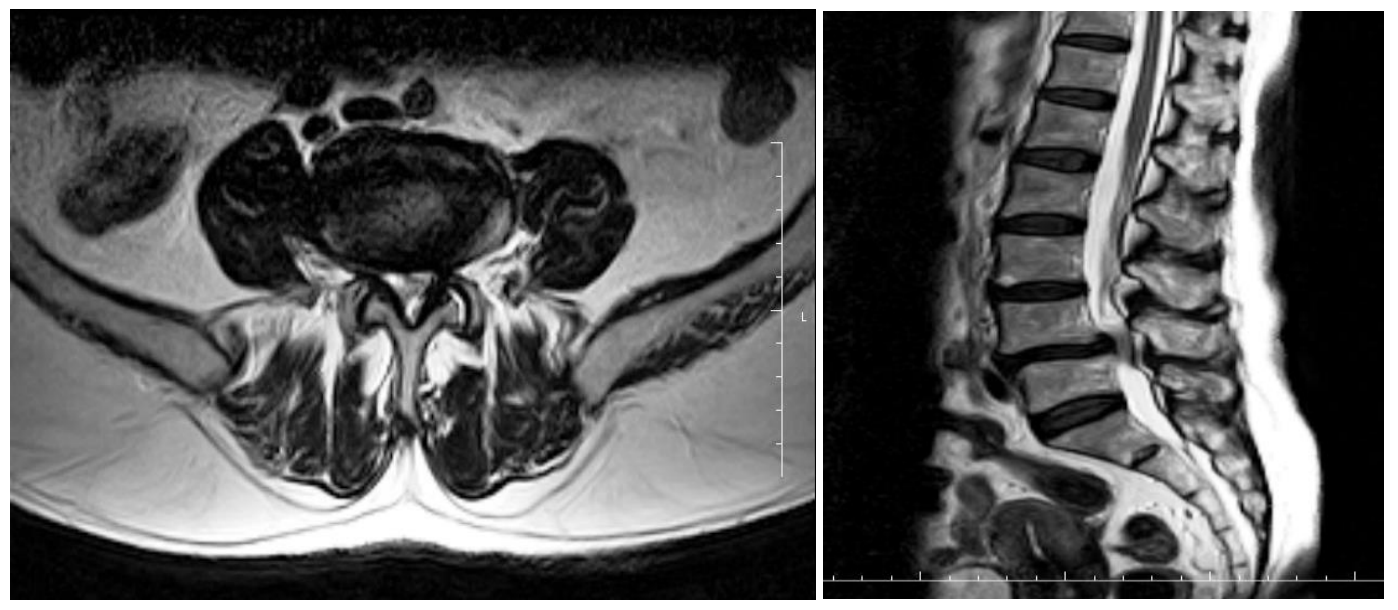

Fig 2. Axial and sagittal sections of stenotic neural canal at L4-5 level

Figure showing angular edged LSS deformity at spinal canal L4-5 level.

Zheng et al. (21) and Singh et al. (12) evaluated the AP diameter of congenital and degenerative LSS compared to a control group. They thought that the pedicule length and vertebral body width in LSS was decreased in the meanwhile AP diameter of vertebral body and body height did not differ when it was compared to the controls. Therefore we mainly focused on the spinal canal diameter measurements in our study.

\section{METHODS}

This study was performed on 81 patients that were treated in Baskent University, Zubeyde Hanim Hospital's Neurosurgery, Physical Therapy and Rehabilitation and Neurology clinics between the years 2011 and 2013. After cardiological examination, ASAPS category I-III (American Society of Anesthesiologists Physical Status) patients with LSS were operated. Medical histories of all patients were taken and they were subjected to a neurological 
examination before and after surgery. The results were evaluated and compared by the JOA scale and postoperative recovery rates were determined as percentages. Images were reviewed by two independent investigators (Radiologist and neurosurgeon).

Demographic parameters such as age and gender were recorded. Anteroposterior radiographs of all patients were taken before and after surgery, lateral hyperflexion and hyperextension positions. PACS software and PACS workstation was used for measurements of posterior protrusion values of herniated disc levels in sagittal sections of lumbar vertebra and also measurements of TIP, M-AP, IF in axial sections.

In our trial; 44 patients have undergone decompressive laminectomy, posterior segmental instrumentation surgery or unilateral laminotomy bilateral decompression. 37 patients have undergone lumbar microdiscectomy surgery.

\section{Statistical Analysis}

SPSS for windows version 17.0 (SPSS. Inc. Chicago, IL, USA) was used for all analysis. Student T-test was used to compare age, JOA scores and bone canal measurements data between groups. Pearson's correlation was used to determine pre and post surgery JOA scores between groups. $\mathrm{P}<0.05$ was considered statistically significant.

The factors of age, gender ratio, initial and 6-12 month JOA score were compared between the two groups LSS and LDH using Student T-test. TIP, M-AP and IF values of LSS and LDH were compared with the T-test.

\section{RESULTS}

Baseline clinical symptoms of patients in our study group are reported in Table 1. 
Table 1: Baseline clinical symptoms of patients

\begin{tabular}{lll}
\hline Patient group & LDH $(N=37)$ & LSS $(N=44)$ \\
\hline Back pain (\%) & 97 & 82 \\
Leg pain (\%) & 91 & 79 \\
Lower extremity motor disability & 8 & 18 \\
$(\%)$ & & \\
Numbness (\%) & 5 & 14 \\
Urinary dysfunction (\%) & 2 & 9 \\
Gait disturbance (\%) & 2 & 14 \\
Neurogenic claudication (\%) & 16 & 52 \\
\hline
\end{tabular}

All measured osteal body diameter mean values (TIP, M-AP, IF) in LSS were less than the LDH group. TIP, M-AP, IF values on both LDH and LSS were compared. The IF mean values were shown to be statistically significant $\mathrm{p}<0.004$. Sagittal disc section measurements were not statistically significant for LSS and LDH $\mathrm{p}<0.182$. The pre and post surgery JOA score mean values of LDH was higher than LSS group $p<0.005$. The recovery ratio 6-12 months after the surgery, JOA score was higher in LDH group $\mathrm{p}<0.001$. According to the surgical procedures, lumbar microsurgery discectomy recovery rate was higher than posterior segmental instrumentation. Surgical recovery rates in LDH and LSS patients was not statistically significant according to the gender parameter $\mathrm{p}<0.93$ (Table 2, Figure 3 ).

Comparison of age and bony structure diameter (Pearson correlation $-0.547, \mathrm{p}<0.001$ ) showed that; MIP, TIP, IF values decreased as the age increased. According to this data, spinal canal diameter was narrower in LSS compared to LDH. TIP -0.513, M-AP -0.352, IF -.573. It was noticed that LSS was seen more frequently in older age compared to LDH, which suggests degenerative process is the main factor in LSS. 
Table 2: Characteristics of 81 patients with JOA scores and spinal canal diameters

\begin{tabular}{|c|c|c|c|c|c|c|c|}
\hline \multirow{2}{*}{$\begin{array}{l}\text { Variable } \\
\text { Age }\end{array}$} & \multicolumn{3}{|c|}{$\begin{array}{l}\text { LDH Group } \\
\mathrm{n}=37\end{array}$} & \multicolumn{3}{|c|}{$\begin{array}{l}\text { LSS Group } \\
\mathrm{n}=44\end{array}$} & \multirow{2}{*}{$\begin{array}{l}\mathbf{P} \\
0,005\end{array}$} \\
\hline & 50,1 & \pm & 14,7 & 66,0 & \pm & 9,8 & \\
\hline TIP (mm) & 20,2 & \pm & 4,0 & 16,4 & \pm & 3,7 & 0,787 \\
\hline $\mathrm{M}-\mathrm{AP}(\mathrm{mm})$ & 14,5 & \pm & 2,8 & 12,9 & \pm & 2,9 & 0,442 \\
\hline $\mathrm{IF}(\mathrm{mm})$ & 16,5 & \pm & 4,1 & 12,6 & \pm & 2,9 & 0,004 \\
\hline Sagittal Disc Plane & 6,6 & \pm & 1,8 & 6,2 & \pm & 1,2 & 0,182 \\
\hline JOA_PreOp & 7,0 & \pm & 1,7 & 6,2 & \pm & 2,9 & 0,005 \\
\hline JOA_6_12_Months & 14,5 & \pm & 1,0 & 13,1 & \pm & 2,1 & 0,004 \\
\hline JOA_Recovery_Rate\% & 93,4 & \pm & 12,1 & 77,7 & \pm & 23,0 & 0,001 \\
\hline
\end{tabular}

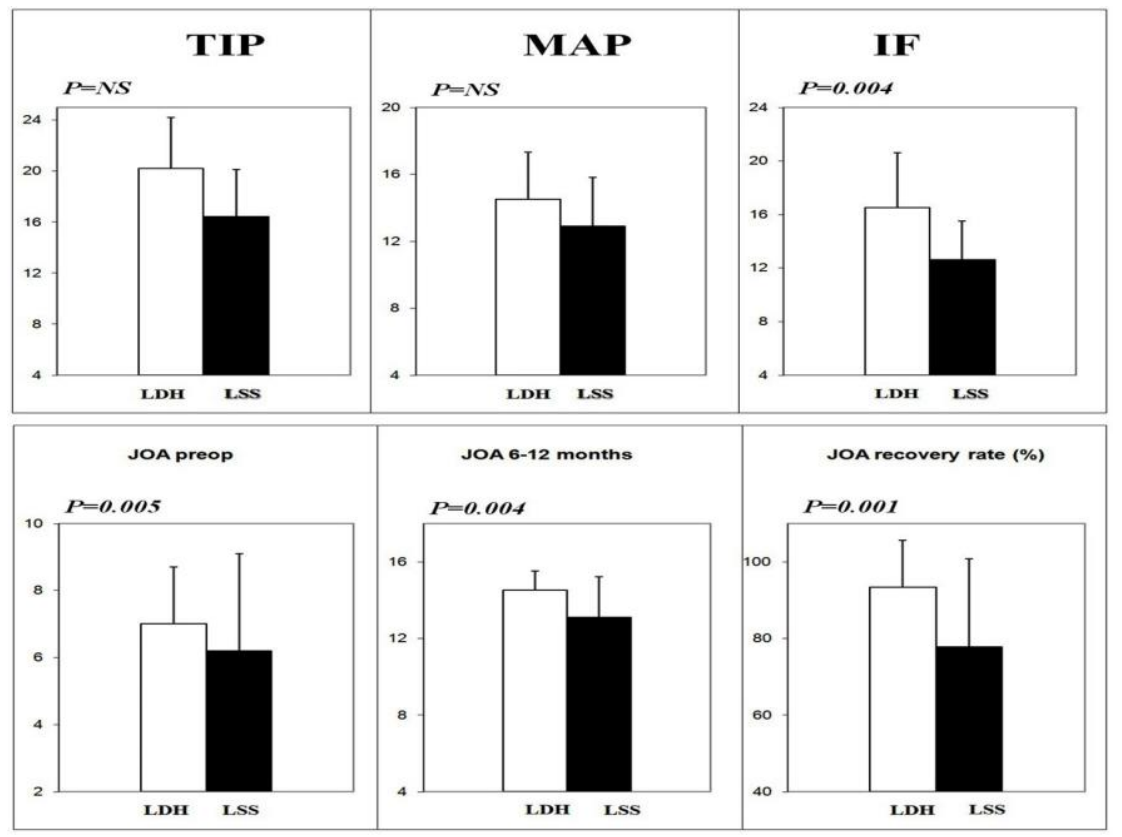

Fig 3: Comparison of spinal canal diameters and JOA scores between LDH and LSS group patients 


\section{DISCUSSION}

Lumbar spinal stenosis is a condition that has different presentations due to the reduction of the total area of spinal canal, lateral recess or neural foramina $(3,7)$. Stenosis develops due to disc degeneration in intervertebral connection points, facet artropathy, ligamentum flavum hypertrophy, spondylosis and sometimes due to the complex process of spondylolisthesis ( 9 , 11). Neurological claudication is the most frequent indication for spinal surgery in individuals diagnosed with lumbar spinal stenosis over 60 years $(2,6,13)$.

The degenerative form of LSS is often limited to a single level of spine, particularly L4-5 $(4,10)$. In contrast the developmental form can be seen in several levels of the spine. In our study, the MRI sections showed that the pathological narrowing of spinal canal is seen mostly at L4-5 in symptomatic patients.

Previous researches indicate that the rate of lumbar spinal stenosis does not change with gender or race. On the other hand, there is literature that shows degenerative LSS is seen at a higher rate with people over 60 and the rate increases with older age (11). Our study also showed no correlation with gender and there was an increase in LSS rate with age. Disc degeneration develops by age and the main factor in degenerative LSS is the combination of other pathologies with disc degeneration. Krikaldy-Willis et al. defined the triple joint complex that occurs in the vertebral disc, upper and lower vertebral body and facet joints (8).

Any change that occurs between the components of this complex affects one another by time. If these changes are severe, they will result in a narrowing of the canal followed by the presentation of clinical symptoms of spinal stenosis. In the current study; we observed that the mean values of TIP, M-AP and IF were less than LDH with LSS. However only the IF values were significant to distinguish LDH and LSS p<0.04; TIP and M-AP values were not statistically significant. This data suggests us that degenerative LSS has a preferential relationship with facet artropathy over other parameters. The deformity of spinal canal from 
ovoid to angular-edged formed by degeneration of facet basis and ligamentum flavum hypertrophy can be explained by the decrease in interfacet diameter which is the main predictor of degenerative LSS in our study.

Neurological symptoms due to compression of dural sac have appeared gradually by the time. Similarly; although lumbar disc herniation and the presence of spondylolisthesis seem like causing the development of lumbar spinal stenosis clinically and radiologically. Sagittal disc section and protruded disc measurements were not statistically significant $p<0.182$ in the current study.

In many studies including Abbas et al. spinal canal measurements were made by computerized tomography. However in our study, ligamentum filavum hypertrophy in narrow spinal canal was also taken under consideration for that reason MRI was used for all measurements (1). Currently MRI has a better spatial resolution and capacity to show soft tissue changes such as ligamentum flavum, disc herniation etc. Preoperative MRI variables and surgical outcomes of patients were compared in a study which two groups had no difference, according to the degree of disc degeneration, foraminal stenosis, facet arthrosis and presence of listhesis $\mathrm{p}<0.05$ (2). We also considered the proposal regarding correlation between surgical outcome and postoperative imaging (MRI or CT scans) of surgically treated LSS patients, but in our study, postoperative imaging was based on radiography. It would be better for future research to consider comparisons between the parameters of postoperative surgical, clinic and imaging changes.

In our study, there were 18 patients with L4-L5 level, 14 patients with L5-S1, 4 patients L3-4 and 1 patient L2-3 level disc hernia and also 30 of the total 81 patients had listhesis. 6 patients were diagnosed with LSS with listhesis a L2-3, L3-4; 18 patients at L4-5; 6 patients at L5-S1. 37 of these patients had undergone lumbar microdiscectomy and 36 of 44 patients had decompressive laminectomy posterior segmental instrumentation performed and 8 of them had 
unilateral laminotomy bilateral decompression by microsurgical technique. In LSS surgery positive results are reported at $80 \%(16,17)$. However in many studies early and mid-term results are good but in long term follow-up results deteriorate and re-stenosis can develop over time $(18,20,22)$. In our study, observations on improvement were made during the postoperative 12 months. We believe that further studies should collect data over a longer term. Turner et al. investigated long-term results after decompression surgery and reported 64\% positive results (14). They also reported that presence of a degenerative spondylolisthesis improves the positive result of the surgery to $85 \%$. It was shown in our study that clinical outcomes of LDH were better than LSS statistically. Also we have a $37 \%$ existence of spondylolisthesis which supports degenerative listhesis is a factor in LSS development.

In Yamashita's study, older and younger patients had similar clinical courses during their observation after surgery. However older patients got worse to a significant extent after 6 months of follow up (19). This study shows us that degenerative processes and neuroischemic changes in advanced age are a predictive factor for prognosis.

Investigating the morphometric characteristics of the lumbar vertebrae in degenerative lumbar spinal stenosis patients can help to establish a more complete model for degenerative LSS pathophysiology and etiology. This can further help in developing useful treatments and improvement of surgery. Furthermore, detection of specific features in an asymptomatic population can be important in prevention or the delay of the symptoms. 


\section{REFERENCES}

1. Abbas J, Hamoud K, May H, Hay O, Medlej B, Masharawi Y, et al. Degenerative lumbar spinal stenosis and lumbar spinal configuration. Eur Spine J 2010;19:1865-73.

2. Alicioglu B, Yilmaz B, Bulakbasi N, Copuroglu C, Yalniz E, Aykac B, et al. Magnetic resonance imaging predictors of surgical outcome in degenerative lumbar spinal stenosis. Jpn J Radiol 2012; 30:811-18.

3. Amundsen T, Weber H, Nordal HJ, Magnaes B, Abdelnoor M, Lilleâs F. Lumbar spinal stenosis: Conservative or surgical management? Spine 2000; 25(11): 1424-36.

4. An HS, Butler JP. Lumbar spinal stenosis: historical perspective, classification, and pathoanatomy. Semin Spine Surg. 1999;11:184-190

5. Arnoldi CC, Brodsky AE, Cauchoix J, Crock HV, Dommisse GF, Edgar MA, et al. Lumbar spinal stenosis and nerve root entrapment syndromes: definition and classification. ClinOrthop 1976;116:4-5,

6. Ciol MA, Deyo RA, Howell E, Kreif S. An assessment of surgery for spinal stenosis: time trends, geographic variations, complications and reoperations. J Am Geriatr Soc 1996;44:285-90.

7. Deen HG Jr, Zimmerman RS, Swanson SK, Larson TR. Assessment of bladder function after lumbar decompressive laminectomy for spinal stenosis: A prospective study. Journal of Neurosurgery 1994; 80: 971-974.

8. Kirkaldy-Willis WH, Wedge JH, Yong-Hing K, Reilly J. Pathology and pathogenesis of lumbar spondylosis and stenosis. Spine 1976; 3:319-28.

9. Pappas CTE, Sonntag VKH. Degenerative disorders of the spine: lumbar stenosis. Menezes AH, Sonntag VKH (Eds), Principles of Spinal Surgery.1996: 631-44.

10. Postacchini F, Cinotti G. Classification and pathomorphology of lumbar stenosis. ChirOrganiMov 1992; 77:7-14. 
11. Schmidek H. Operative neurosurgical techniques: Indications, methods and results, 4th edition, Saunders Company, Massachusetts 2000:2207-17.

12. Singh K, Samartzis D, Vaccaro AR, Nassr A, Andersson GB, Yoon ST, et al. Congenital lumbar spinal stenosis: a prospective, control-matched, cohort radiographic analysis. Spine J 2005;5:615-22.

13. Szpalski M, Gunzburg R. The role of surgery in the management of low back pain. Baillieres Clin Rheumatol 1998;12:141-59.

14. Turner JA, Ersek M, Herron L, Deyo R Surgery for lumbar spinal stenosis. Attempted meta-analysis of the literature. Spine 1992;17(1):1-8.

15. Weinstein PR, Ciricillo SF. Lumbar Spinal Stenosis and Lateral Recess Syndrome. In Rengachary SS, Wilkins RH (Eds), Principles of Neurosurgery. Wolfe 1993: 46.246.18 .

16. Westmark RM, Weinstain PR. Surgical management of lumbar spinal stenosis: Operative Neurosurgical Techniques, WB Saunders Com. Philadelphia 1995:1957-64.

17. White AA, Panjabi MM. Clinical Biomechanics of the Spine, 2nd ed. Philadelphia. PA. Lippincott, 1990.

18. Wilkins RH. Lumbar intervertebral disc herniation. Principles of Neurosurgery. 1994:45.2-45.9.

19. Yamashita K, Ohzono K, Hiroshima K. Five-year outcomes of surgical treatment for degenerative lumbar spinal stenosis. Spine 2006; 31:1484-90.

20. Zheng F, Cammisa FP Jr, Sandhu HS, Girardi FP, Khan SN. Factors predicting hospital stay, operative time, blood loss and transfusion in patients undergoing revision posterior lumbar spine decompression fusion and segmental instrumentation. Spine 2002; 27(8): 818-24. 
21. Zheng F, Farmer JC, Sandhu HS, O'Leary PF. A novel method for quantitative evaluation of lumbar spinal stenosis. HSS J 2006;2:136-40.

22. Zileli M, Özer F. Omurilik ve Omurga Cerrahisi, cilt 1, Meta Basım Matbaacılık Hizmetleri, İzmir, 2002: 739-46. 